\title{
Kruskal-Wallis Test
}

National Cancer Institute

\section{Source}

National Cancer Institute. Kruskal-Wallis Test. NCI Thesaurus. Code C53248.

A nonparametric test for finding if three or more independent samples come from

populations having the same distribution. It is a nonparametric version of ANOVA. 